Bio - grafia. Escritos sobre la Biología y su Enseñanza. ISSN 2027

Edición Extraordinaria. p.p. 785 - 798

Memorias del VIII Encuentro Nacional de Experiencias en Enseñanza de la Biología y la Educación Ambiental. III Congreso Nacional de Investigación en Enseñanza de la Biología.

\title{
ANÁLISIS DEL ESTADO ACTUAL DE LA ENSEÑANZA DE LOS GRUPOS SANGUÍNEO
}

\section{ANALYSIS OF CURRENT STATUS OF TEACHING OF BLOOD GROUPS}

Palacio Muñoz, C. A.

Licenciado en Biología De La Universidad Distrital Francisco José De Caldas, Estudiante de Maestría en Educación con Énfasis Educación en Ciencias de la Naturaleza y la Tecnología. Grupo de Investigación BER (Biología, Enseñanza y Realidades)

Resumen: El presente artículo realiza un acercamiento al estado actual de la enseñanza del concepto de los grupos sanguíneos a partir de la revisión documental de cuatro documentos previamente seleccionados de las bases de datos ERIC, Dialnet y SciELO, resultado de la construcción de los parámetros de selección a la hora de la aplicación de la herramienta bibliométrica, y estudiados desde las categorías de análisis de: las concepciones para la construcción de las narraciones históricas del concepto o metodologías utilizadas en la enseñanza del mismo, la relación con conceptos asociados, el uso del laboratorio, los tipos de enfoques utilizados en la enseñanza de las ciencias, el uso de las tecnologías de la información y la comunicación (TIC) en la enseñanza y por último, el rol del maestro en el proceso enseñanza aprendizaje, abordando de este modo distintas dimensiones y percepciones que se dan frente a este proceso de enseñanza-aprendizaje, siendo este un concepto utilizado habitualmente en la vida diaria, como en el caso de las historias clínicas o por el contrario en escenarios de educación superior en dónde predomina las investigaciones enfocadas al estudio de enfermedades, estudios estadísticos poblacionales en relación con enfoques didácticos de su enseñanza.

Palabras claves: grupo sanguíneo, enseñanza, historia, clases de aprendizaje, rol del maestro, aprendizaje experiencial, TIC 
Edición Extraordinaria. p.p. $785-798$

Memorias del VIII Encuentro Nacional de Experiencias en Enseñanza de la Biología y la Educación Ambiental. III Congreso Nacional de Investigación en Enseñanza de la Biología.

\begin{abstract}
:
This article makes an approach to the current states of teaching the concept of blood groups from the documental review of four preselected documents taken from data bases such as ERIC, Dialnet and SciELO resulting from the construction of the selection parameters to When applying bibliometric tools, and studied from the categories of analysis: the concepts for the construction of historical narratives of the concept or methodologies used in the education of the same, Regarding associated concepts, compared to use of laboratorios, the types of approaches used in science education, the use of Information and Communications Technology (ICT) in teaching and finally the role of the teacher in the teaching-learning process, thereby addressing different dimensions and perceptions that exist against this process of teaching and learning, this being a concept commonly used in daily life, as in the case of medical records or otherwise higher education scenarios where research focused on the study of diseases, population statistical studies regarding its predominant teaching education approaches.
\end{abstract}

Keywords:blood type, education, history, types of learning, teacher's role, experiential learning, TIC. 
Bio - grafia. Escritos sobre la Biología y su Enseñanza. ISSN 2027

Edición Extraordinaria. p.p. 798- 811

Memorias del VIII Encuentro Nacional de Experiencias en Enseñanza de la Biología y la Educación Ambiental. III Congreso Nacional de Investigación en Enseñanza de la Biología.

\section{INTRODUCCIÓN}

El estudio que dio origen al descubrimiento de los grupos sanguíneos lo propuso Karl Landsteiner en 1900, a partir de la observación de las reacciones aglutinación que se presentaban en la mezcla de sueros y células sanguíneas entre individuos en los procesos de transfusión sanguínea (TS), que para ese tiempo eran consideradas peligrosas por lo antecedentes en el tratamiento del sujeto Antoine Mauroy de 34 años, quien perdió la vida al realizársele TS por Jean Denis a mediados de siglo XVII (Pérez, 2009). Siendo este procedimiento analizado o propuesto desde la dinámica circulatoria William Harvey en donde el movimiento de la sangre se describe como un fenómeno puramente físicomecánico. (González, 1995) que en sí, no solo bastó para determinar el éxito de esta técnica médica.

Si bien, existían casos en donde la TS tenía éxito, no solo entre organismos de la misma especie y entre diferentes especies, Blundem propuso indicaciones y contraindicaciones sobre esta técnica, puesto que aumentaba el riesgo de mortalidad en organismos de diferentes especies. (Jaime, 2009)

De este modo, el experimento propuesto por Landsteiner sobre las muestras de sangre que fueron proporcionadas por sus colegas de laboratorio, fueron utilizadas para determinar si se presentaba una aglutinación o no, entre los componentes del suero y los glóbulos rojos intercambiados de cada muestra, demostró que solo en algunos casos se producía la aglutinación, por lo que infirió que existen diferentes categorías en las que se pueden agrupar los muestras de sangre. (Curtis, Barnes, Schnek, \& Massarini, 2008) De esta manera, se nombra a sus primeros hallazgos como el sistema $A B O$, debido a que solo logra reconocer tres distintos tipos de sangre, poco más de un año Sturle y Decastello compañeros de Landsteiner descubrieron un cuarto grupo, el cual presentaba características de dos grupos a la vez y al cual se le designo AB. ¿Pero porque los glóbulos rojos se aglutinan?, este fenómeno es explicado a partir de la membrana celular y las proteínas que están presentes en los eritrocitos, nombrados como antígenos $A$ y $B$, mientras que en el plasma sanguíneo se encuentran los anticuerpos que pueden contener anti-A o anti-B según el caso del tipo de antígeno que presente su eritrocito. Mientras que para los casos de los grupos sanguíneos $O$ y $A B$; el primero presenta anti- $A$ y anti- $B$ en el plasma sanguíneo, y por el contrario el grupo $A B$ presenta proteínas $A$ y $B$ en la membrana de sus eritrocitos. (Arbeláez, 2009). 
Bio - grafia. Escritos sobre la Biología y su Enseñanza. ISSN 2027

Edición Extraordinaria. p.p. $798-811$

Memorias del VIII Encuentro Nacional de Experiencias en Enseñanza de la Biología y la Educación Ambiental. III Congreso Nacional de Investigación en Enseñanza de la Biología.

De acuerdo con la construcción histórica, del descubrimiento de los grupos sanguíneos se propició una nueva era en el campo de la medicina general, transfusional, inmunológica haciendo más relevante la investigación y la formación en este campo (Jaime, 2009).

En consecuencia, la revisión documental sobre la enseñanza de este concepto se centró en las categorías de narraciones históricas, enfoques en la enseñanza de las ciencias, uso de TIC en la enseñanza y por último el rol del maestro.

\section{Frente a las narraciones históricas}

Una de las metodologías que tomaron fuerza en el siglo XX, fue la incorporación de la narración histórica dentro de la investigación de la biología, (Jiménez, 2003), y más aún en la enseñanza de la misma; no obstante el papel que juega el investigador a la hora de generar el relato es condicionado por su propia mirada, debido a que este mismo selecciona, ordena y acomoda los acontecimientos de acuerdo al énfasis que quiere proporcionar a la hora de abordar un tema (Lombardi, 1997). De este modo, se plantean dos tipos de miradas de la historia en la ciencia, una desde el internalismo que se enmarca en que la producción científica está libre de los agentes externos del contexto situándola solo en el marco de las ideas y filosofías que atañen al investigador en su momento o por el contrario externalistas en donde otras disciplinas y ciencias aportan a la construcción histórica de cada uno de los pensadores de ese tiempo, generando una construcción contextualizada a las fluctuaciones de ese hito histórico. (Medina, 1983).

\section{En relación con conceptos asociados}

Otra de las formas de abordar el concepto de grupos sanguíneos surge de la relación con la temática hereditaria, en donde este se posiciona como un ejercicio práctico para el desarrollo de explicaciones como la codominacia, enfermedades hemolíticas por incompatibilidad de los grupos sanguíneos y factor Rhesus (Rh), mientras que en otras surgen en relación con los protocolos y técnicas de procedimientos para las transfusiones sanguíneas, pruebas de paternidad, entre otras.

\section{Frente al uso de laboratorios físicos}

El uso del laboratorio en el proceso enseñanza- aprendizaje de las ciencias se ha utilizado habitualmente como una estrategia de relación entre lo teórico y lo práctico, debido a que se centra en actividades que tienden a verificar, comprobar y justificar planteamientos 0 teorías ya preestablecidas acercando al estudiante a construir de forma mecánica, las destrezas y los hábitos propios de un trabajo en ciencias. (Kirschner.1992, citado por Caballero, M; Flores, J; Moreira, M. 2009), al igual que lo plantea Osorio (2004 citado por López, A; Tamayo, O. 2012) cuando se refiere a la actividad experimental, no solo desde 
Bio - grafia. Escritos sobre la Biología y su Enseñanza. ISSN 2027

Edición Extraordinaria. p.p. $798-811$

Memorias del VIII Encuentro Nacional de Experiencias en Enseñanza de la Biología y la Educación Ambiental. III Congreso Nacional de Investigación en Enseñanza de la Biología.

una visión instrumental, sino como un ambiente de aprendizaje que promueve objetivos conceptuales, procedimentales y actitudinales en los estudiantes.

De este modo, las prácticas de laboratorio pueden clasificarse desde la perspectiva de Caballer y Oñorbe (1999 citado por López, A; Tamayo, O. 2012) como: problemascuestiones (pretende es comprobar teorías), problemas-ejercicio (reforzar el aprendizaje de las técnicas preestablecidas) y problemas-investigación (reconocer y aplicar metodologías propias de la investigación).

\section{Frente a los enfoques de la enseñanza en ciencias}

Las distintas perspectivas que se han abordado frente a la enseñanza de las ciencias corresponden a los distintos enfoques que orientan la práctica docente. De este modo, para Pozo (1997) describe cada uno de los enfoques de la enseñanza de las ciencias a partir de tres relaciones como lo son: supuestos epistemológicos y la concepción de aprendizaje, selección y organización de los contenidos, enseñanza y evaluación, como lo explica Pozo (1997) a continuación:

> Tradicional: la lógica de la disciplina como un conjunto de hechos, entre las actividades más comunes es la transmisión oral, y posee relación con el realismo interpretativo.

> Descubrimiento: metodología científica como lógica de la disciplina, entre las actividades más comunes son la investigación y el descubrimiento, y posee relación con el constructivismo.

- Expositiva: la lógica de la disciplina como sistema conceptual, entre las actividades más comunes es la enseñanza por exposición, y posee relación con el constructivismo.

$>$ Conflicto cognitivo: la lógica de la disciplina y los conocimientos previos, entre las actividades más comunes cambios de los conocimientos previos, y posee relación con el constructivismo.

> Investigación: la lógica de la disciplina como solución de problemas, entre las actividades más comunes es la enseñanza a través de la resolución de problemas, y posee relación con el constructivismo.

> Modelos: los contenidos como vía para construir modelos, entre las actividades más comunes es la enseñanza explicación y reestructuración de los modelos, y posee relación con el realismo interpretativo.

Frente al uso de las TIC en el proceso de enseñanza

En la actualidad el uso de las TIC, ha venido tomando mayor fuerza dentro de los programas educativos principalmente en primaria y secundaria, estimulado por las políticas educativas para la calidad de la educación. (Coll, Mauri, \& Onrubia, 2004), por otro lado, el continuo uso de las TIC, han llevado a que se genere una clasificación de acuerdo a la 
Bio - grafia. Escritos sobre la Biología y su Enseñanza. ISSN 2027

Edición Extraordinaria. p.p. 798- 811

Memorias del VIII Encuentro Nacional de Experiencias en Enseñanza de la Biología y la Educación Ambiental. III Congreso Nacional de Investigación en Enseñanza de la Biología.

relación del instrumento mediador entre alumno y contenido, entre profesor y contenido, entre alumno y profesor, entre la actividad desplegada por los profesores y los alumnos en actividades de enseñanza aprendizaje, o como un instrumento configurador del espacio y el aprendizaje. (Coll, Mauri, \& Onrubia, 2004)

Frente al rol del profesor

Con relación a las formas de enseñar el maestro juega un papel importante en concordancia con las acciones, métodos y enfoques que se han descrito en la categoría de enfoques. De este modo, se hace necesario describir el rol que juega en la dinámica del proceso enseñanza aprendizaje, ya sea como orientador de la práctica, transmisionista, agente estimulador de los cambios de ideas previas, formulador de conflictos y guía de su solución, creador de discusiones polémicas en pro de que estudiante elabora su propia solución (Pozo, 1997).

\section{METODOLOGÍA}

La propuesta metodológica utilizada para la creación de este artículo fue dividida en tres fases que corresponden a las siguientes:

I. La fase bibliométrica, de orden preparatorio, representa el procedimiento de búsqueda y recopilación de fuentes de información según su naturaleza y características; es la aproximación al objeto de estudio, a través de la delimitación y definición de estrategias particulares de búsqueda (Barbosa Chacón, Barbosa Herrera, \& Rodríguez Villabona, 2013, p. 90) como lo son: idioma: español portugués e inglés, con una ventada de búsqueda entre 2005-2015, y palabras claves: grupo sanguíneo, tipos de sangre, antígenos sanguíneos, anticuerpo sanguíneo, herencia de grupo sanguíneo, enseñanza.

II. Fase de categorización se construyó con base a las relaciones en aspectos metodológicos de la enseñanza en ciencia y papel del maestro, construidas desde la perspectiva de varios autores.

III. Fase de interpretación en donde cada documento que fue seleccionado de la fase bibliométrica es contrastado con las categorías construidas. 
Bio - grafia. Escritos sobre la Biología y su Enseñanza. ISSN 2027

Edición Extraordinaria. p.p. 798- 811

Memorias del VIII Encuentro Nacional de Experiencias en Enseñanza de la Biología y la Educación Ambiental. III Congreso Nacional de Investigación en Enseñanza de la Biología.

\section{RESULTADOS}

De acuerdo a los criterios de búsqueda, se obtuvo 1288 artículos que fueron registrados en la tabla 1 y en donde un $27 \%$ de la documentación pertenecía a la base de datos ERIC, $25 \%$ a la base ScIELO y un $48 \%$ a Dialnet, siendo este ultima con mayor número de publicaciones en relación al concepto de grupos sanguíneos.

\begin{tabular}{lccccc}
\hline & \multicolumn{3}{c}{ BASES DE DATOS } & \\
\cline { 2 - 4 } \multicolumn{1}{c}{ CONCEPTOS CLAVES } & ERIC & SciELO & Dialnet & TOTAL \\
\hline Grupos sanguíneos & 217 & 194 & 200 & 611 \\
Tipos de sangre & 78 & 86 & 341 & 505 \\
Antígenos sanguíneos & 2 & 18 & 2 & 22 \\
Anticuerpos sanguíneos & 4 & 20 & 46 & 70 \\
Herencia grupos sanguíneos & 50 & 2 & 28 & 80 \\
TOTAL & 351 & 320 & 617 & 1288 \\
PROMEDIO & $27 \%$ & $25 \%$ & $48 \%$ & $100 \%$ \\
\hline
\end{tabular}

Tabla 1. Criterios de búsqueda inicial

De acuerdo a los criterios de exclusión, se descartaron 1284 artículos debido a la relación con estudios e investigaciones demográficas y médicas fuera del campo educativo, mientras que los criterios de inclusión permitieron caracterizar su naturaleza dentro del campo de la enseñanza, como lo demuestra la siguiente tabla:

\section{Criterios de selección}

Inclusión Relación con la enseñanza

Relación con la historia concepto

Relación con instrumentos 1 tecnológicos y/o ambientes de aprendizaje

Relación con la enseñanza con de la genética y la herencia

Total de documentos incluidos

\section{Exclusión}

estadísticos

poblacionales

Estudios disciplinares aplicadas

\section{Bases de datos

ERIC SCIELO Dialnet

\section{Total}

0

0

1

0

1

$1 \quad 0 \quad 1$

0

$0 \quad 1$

1

1

0

1

0

$\begin{array}{ccc} & & \\ & 4\end{array}$

28

17

68

292

$600 \quad 1219$


Bio - grafia. Escritos sobre la Biología y su Enseñanza. ISSN 2027

Edición Extraordinaria. p.p. 798- 811

Memorias del VIII Encuentro Nacional de Experiencias en Enseñanza de la Biología y la Educación Ambiental. III Congreso Nacional de Investigación en Enseñanza de la Biología.

a enfermedades

Total de documentos excluidos

1284

Tabla 2. Criterios de inclusión y exclusión de artículos

Por otro lado, los criterios de inclusión referidos a la enseñanza, historia del concepto, tecnologías y relacionados a los campos de la herencia y genética, arrojo como resultado cuatro artículos presentes en las bases de datos ya nombradas, al igual que se adicionaron dos artículos más del portal búsqueda de Google académico descritos a continuación:

\begin{tabular}{|c|c|c|c|c|c|c|c|}
\hline ARTÍCULO & REVISTA & $\begin{array}{l}\text { BASE } \\
\text { DE } \\
\text { DATO } \\
S\end{array}$ & $\begin{array}{l}\text { AUTOR } \\
\text { (ES) }\end{array}$ & $\begin{array}{c}\text { ISS } \\
\mathrm{N}\end{array}$ & $\begin{array}{c}A \tilde{N} \\
0\end{array}$ & $\begin{array}{c}\text { Vol } \\
\text {. }\end{array}$ & No. \\
\hline $\begin{array}{l}\text { Evolución del } \\
\text { conocimiento sobre la } \\
\text { sangre y su } \\
\text { movimiento, parte II }\end{array}$ & $\begin{array}{l}\text { Revista } \\
\text { de } \\
\text { investigac } \\
\text { ión clínica }\end{array}$ & $\begin{array}{l}\text { SciEL } \\
0\end{array}$ & $\begin{array}{l}\text { Michelli, } \\
\text { A; } \\
\text { Sierra, }\end{array}$ & $\begin{array}{c}0034 \\
- \\
8376\end{array}$ & $\begin{array}{c}200 \\
5\end{array}$ & 57 & 1 \\
\hline $\begin{array}{l}\text { El espacio europeo de } \\
\text { educación } \\
\text { superior(EES) y su } \\
\text { repercusión en los } \\
\text { procesos enseñanza } \\
\text { aprendizaje de la } \\
\text { inmunología }\end{array}$ & $\begin{array}{l}\text { Revista } \\
\text { de } \\
\text { enseñanza } \\
\text { universita } \\
\text { ria }\end{array}$ & $\begin{array}{l}\text { Dialne } \\
+\end{array}$ & $\begin{array}{l}\text { Maldona } \\
\text { do, D. }\end{array}$ & $\begin{array}{l}1131- \\
5245\end{array}$ & $\begin{array}{c}200 \\
7\end{array}$ & & 29 \\
\hline $\begin{array}{l}\text { Sciencie in drama: } \\
\text { using television } \\
\text { programmes to teach } \\
\text { concepts } \\
\text { teachniques }\end{array}$ & $\begin{array}{l}\text { School } \\
\text { Science } \\
\text { Review }\end{array}$ & ERIC & $\begin{array}{l}\text { Rutter, } \\
\text { G. }\end{array}$ & $\begin{array}{l}0036 \\
-6811\end{array}$ & $\begin{array}{c}201 \\
1\end{array}$ & 92 & 341 \\
\hline $\begin{array}{l}\text { Grupos sanguíneos y } \\
\text { enfermedad }\end{array}$ & $\begin{array}{l}\text { Medicina } \\
\text { clínica }\end{array}$ & $\begin{array}{l}\text { Dialne } \\
t\end{array}$ & $\begin{array}{l}\text { Gonzále } \\
z, A \text {. }\end{array}$ & $\begin{array}{c}0025 \\
- \\
7753\end{array}$ & $\begin{array}{c}200 \\
5\end{array}$ & 125 & 10 \\
\hline
\end{tabular}


Bio - grafia. Escritos sobre la Biología y su Enseñanza. ISSN 2027

Edición Extraordinaria. p.p. 798-811

Memorias del VIII Encuentro Nacional de Experiencias en Enseñanza de la Biología y la Educación Ambiental. III Congreso Nacional de Investigación en Enseñanza de la Biología.

\begin{tabular}{|c|c|c|c|c|c|c|c|}
\hline $\begin{array}{l}\text { El modelo bandler- } \\
\text { grinder de aprendizaje } \\
\text { y la enseñanza de la } \\
\text { genética mendeliana en } \\
\text { estudiantes } \\
\text { costarricenses de } \\
\text { décimo año. }\end{array}$ & $\begin{array}{l}\text { Research } \\
\text { Journal } \\
\text { of the } \\
\text { Costa } \\
\text { Rica } \\
\text { distance } \\
\text { education } \\
\text { universita } \\
\text { ry }\end{array}$ & $\begin{array}{l}\text { Portal } \\
\text { Google } \\
\text { acadé } \\
\text { mico }\end{array}$ & $\begin{array}{l}\text { Chavarrí } \\
\text { a, S. } \\
\text { Bermúd } \\
\text { ez, T. } \\
\text { Villalobo } \\
\text { S, N } \\
\text { Morera, } \\
\text { B. }\end{array}$ & $\begin{array}{c}1659 \\
- \\
4266\end{array}$ & $\begin{array}{c}201 \\
2\end{array}$ & 4 & 2 \\
\hline $\begin{array}{l}\text { A abordagem histórica } \\
\text { do sistema de grupo } \\
\text { sanguíneo } A B O \text { nos } \\
\text { livros didáticos de } \\
\text { ciências e biología }\end{array}$ & $\begin{array}{l}\text { Filosofia } \\
\text { e História } \\
\text { da } \\
\text { Biologia }\end{array}$ & $\begin{array}{l}\text { Portal } \\
\text { Google } \\
\text { acadé } \\
\text { mico }\end{array}$ & $\begin{array}{l}\text { Belotto, } \\
\text { C: } \\
\text { Caluzi, } \\
\text { J. } \\
\text { Nabuco, } \\
\text { E. } \\
\text { Guardia } \\
\text { no, S }\end{array}$ & - & $\begin{array}{c}200 \\
7\end{array}$ & 2 & - \\
\hline
\end{tabular}

Tabla 3.articulos seleccionados para la revisión

\section{DISCUSIÓN}

Frente a las categorías y artículos seleccionados, se obtuvo el siguiente análisis:

\section{Narración histórica}

Los autores que describen una historiografía internalista son: González, A. (2005) debido a que su construcción histórica solo se basa en las condiciones en que evoluciona el pensamiento cientificista, sin contemplar las fluctuaciones del contexto, que dieron cabida a la evolución de este, y por el contrario se describe dicha evolución como la suma de acontecimientos que dieron como origen la idea actual del concepto, como lo explica Medina (1983): "El internalismo asume que los procesos de producción y validación del conocimiento están libres de influencias externas, y su estudio compete únicamente a la historia de las ideas y a la filosofía de la ciencia."(p.55), asimismo, Belotto, C. Caluzi, J. Nabuco, E. \& Guardiano, S. (2007), establecen una relación importante sobre la construcción histórica de varios libros acerca del concepto, aludiendo que son muy pocos las historiografias armadas 
Bio - grafia. Escritos sobre la Biología y su Enseñanza. ISSN 2027

Edición Extraordinaria. p.p. 798- 811

Memorias del VIII Encuentro Nacional de Experiencias en Enseñanza de la Biología y la Educación Ambiental. III Congreso Nacional de Investigación en Enseñanza de la Biología.

con datos de primera fuente como lo son libros $y / 0$ documentos propios de los investigadores que determinaron las características de estos grupos sanguíneos; por otro lado, los otros artículos no presentan narraciones históricas del concepto, ni la metodología.

\section{En relación con conceptos asociados}

En el artículo del modelo de Bandler-Grinder, el concepto de grupos sanguíneos se utiliza para explicar la herencia de tipos de sangre, convirtiéndolo en una situación problema para explicar la combinación de alelos, la dominancia y dominancia incompleta entre caracteres hereditarios, en el cual los estudiantes ponen a prueba los conocimientos sobre la aplicación de las leyes de Mendel sin llegar a profundizar en el tema de los grupos sanguíneos; por otra parte, Maldonado (2007) y Rutter (2011) utilizan este concepto para afianzar o validar técnicas y metodologías utilizadas para procedimientos clínicos para el caso de las transfusiones sanguíneas y otra para el reconocimiento de las características propias de la parte experimental de una investigación.

\section{Frente al uso de laboratorios}

En este sentido, solo dos de los artículos presentan el trabajo experimental, Maldonado (2007) y Rutter (2011), en donde el primero plantea la práctica experimental como un recetario de instrucciones con el ánimo de fomentar o validar las técnicas y teorías con las cuales se debe llevar acabo la extracción o caracterización de los grupos sanguíneos para los procedimientos transfusionales del tejido sanguíneo, tomando de esta forma un enfoque de las prácticas como problemas-ejercicio, en donde se pretende validar y mecanizar una técnica, al igual que se utiliza para concluir un tema específico, (Caamaño, 1992, 2003 citado por López, et Al 2012); mientras que el segundo lo plantea como estructura básica para fomentar la investigación posicionándolo como una práctica Problemas-investigación, de este modo, se genera un aprendizaje significativo, como lo explica Tenreiro y Vieira (2006 citado por López, et Al 2012) cuando explica que los laboratorios como investigación construyen nuevos conocimientos conceptuales en el ámbito de la resolución de problemas. 
Bio - grafia. Escritos sobre la Biología y su Enseñanza. ISSN 2027

Edición Extraordinaria. p.p. 798- 811

Memorias del VIII Encuentro Nacional de Experiencias en Enseñanza de la Biología y la Educación Ambiental. III Congreso Nacional de Investigación en Enseñanza de la Biología.

\section{Enfoques de la enseñanza en ciencias}

El articulo de Maldonado, D. (2007), el enfoque que prevaleció fue la enseñanza expositiva debido a que se mantenían parte clases magistrales para el acercamiento a la terminología científica, partiendo de las propias concepciones de los estudiantes, cuando describen que se hacían aclaraciones de los conceptos científicos luego de generar el debate desde sus propios imaginarios y aterrizándolos con propio lenguaje de las ciencias, igual que lo plantea Pozo (1997) "parece asumir asimismo que ese acercamiento entre el significado psicológico y el lógico requiere un cierto paralelismo entre las estructuras conceptuales del alumno y las estructuras de conocimiento científico, de forma que su acercamiento progresivo"(p.281); las metodologías utilizadas fueron de carácter teórico-práctico, en donde sobresale como producto terminado las exposiciones de los estudiantes.

Por otro lado, Chavarría, S. Bermúdez, T. Villalobos, N. \& Morera, B.(2012), mantienen un enfoque tradicional en la forma en como enseñaban las dos maestras dentro del estudio realizado, debido al poco bagaje en estrategias para abordar el tema de la herencia de los grupos sanguíneos, proponiendo estrategias guiadas únicamente entre lo explicado y lo evaluado.

Y por último Rutter, G. (2011), propone desde un solo programa de televisión explicar diferentes técnicas de laboratorio para tipificar aspectos como grupos sanguíneos a través de la resolución de problemas para ir descartando al criminal en el homicidio de señor Burns, por lo que el enfoque se trabaja es de investigación dirigida, en donde el estudiante asume el papel de investigador para resolver el crimen y por lo tanto utilizara métodos y procedimientos propios de una investigación científica.

\section{Uso de las TIC en el proceso de enseñanza}

Al respecto, sólo dos artículos demostraron la aplicación y la necesidad de utilizar estas herramientas, por un lado, Maldonado, D.(2007) utilizó la categoría de instrumento mediador entre alumno y contenido, debido a que se le plantean al estudiante utilizar bases 
Bio - grafia. Escritos sobre la Biología y su Enseñanza. ISSN 2027

Edición Extraordinaria. p.p. $798-811$

Memorias del VIII Encuentro Nacional de Experiencias en Enseñanza de la Biología y la Educación Ambiental. III Congreso Nacional de Investigación en Enseñanza de la Biología.

de datos y portales de información para su propia elaboración del conocimiento, como lo explican Coll, Mauri, \& Onrubia (2004), al utilizar un instrumento mediador entre profesor y contenido, al momento que se emplean las ayudas audiovisuales para complementar sus explicaciones; por otro lado, el artículo de Rutter, G. (2011), solo utilizó la relación profesor-contenido al explicar cómo las técnicas pueden describirse desde el mismo programa animado de los Simpsom.

\section{Rol del profesor}

En relación con los artículos ya descritos por sus enfoques y en el orden en que se establecen en esa categoría, el primero de estos, posee un enfoque expositivo y el papel que juega el maestro resulta ser activo debido a que guía y dirige cada una de las fases de organización del trabajo, explica y organiza el lenguaje promoviendo mirada crítica del conocimiento tomando como mayor fuerza el poder explicativo por parte de este.(Pozo, 1997):mientras que el segundo artículo descrito en el mismo se establece al profesor desde un enfoque tradicional operando de esta forma por parte de las profesoras un papel transmicionista en el aula, reproduciendo o replicando información y ejercicios descontextualizados para los estudiantes, y por último en el papel que juega el profesor del enfoque investigación dirigida a través de un programa de televisión Los Simpsom, el maestro desarrollo un papel generador de conflictos, como a su vez, de orientador de soluciones de estos posicionándolo con un carácter cuestionador de dichas soluciones.

\section{CONCLUSIONES}

La tendencia de las narraciones históricas del concepto de grupos sanguíneos dentro del periodo analizado (2005-2015), poseen características propias del internalismo descritas por Medina (1983) al dejar de lado el contexto socio-económico, cultural en que se da evolución del concepto, al igual que dichas reconstrucciones históricas se elaboran a partir de otras reconstrucciones.

Los enfoques en las enseñanzas de las ciencias no son considerados como buenos o malos, por el contrario demuestran si las practicas que se están abordando van de acuerdo a la población de estudiantes del aula de clase (Pozo, 1997).

El uso de las TIC como instrumento mediador entre el profesor y el contenido tiende a ser el más utilizado, por ser más afín a la direccionalidad del que guía el proceso de 
Bio - grafia. Escritos sobre la Biología y su Enseñanza. ISSN 2027

Edición Extraordinaria. p.p. 798- 811

Memorias del VIII Encuentro Nacional de Experiencias en Enseñanza de la Biología y la Educación Ambiental. III Congreso Nacional de Investigación en Enseñanza de la Biología.

aprendizaje convirtiéndolo solo en un accesorio de la clase para mantener atentos a los estudiantes y no como un propiciador de conocimiento frente a este concepto.

La producción de artículos sobre la enseñanza del concepto es mínima a comparación de las producciones en investigación en relación con el concepto de grupo sanguíneo como técnica para estudios de enfermedades o estadísticos poblacionales como se demostró en el rastreo bibliométrico.

El rol del profesor tiende a ser cada vez más apático al papel transmisionista y toma fuerza el desempeño de orientador, ofreciéndole mayor protagonismo al estudiante en su proceso de enseñanza aprendizaje, convirtiéndose a su vez en mediador entre el conocimiento y el estudiante, por lo que es necesario replantear las prácticas de laboratorio en relación a la construcción de explicaciones por parte de los estudiantes que coadyuven a la comprensión de fenómenos en los que se ven integrados conocimientos como los grupos sanguíneos.

\section{REFERENCIAS BIBLIOGRAFICAS}

Arbeláez. C. A. (2009). Sistema de grupo sanguíneo ABO. Medicina \& Laboratorio, 15(7-8), 329-349.

Barbosa Chacón, J. W., Barbosa Herrera, J. C., \& Rodríguez, M. (2013). Revisión y análisis documental para estado del arte: una propuesta metodológica desde el contexto de la sistematización de experiencias educativas. INVESTIGACIÓN BIBLIOTECOLÓGICA, 27(61), 83-105.

Coll, C., Mauri, T., \& Onrubia, J. (2004). La utilización de las tecnologías de la información y la comunicación en la educación: Del diseño tecno-pedagógico a las prácticas. En Psicología de la educación virtual (75-103). Madrid: Ediciones Morata S.L.

Curtis, H., Barnes, S., Schnek, A., \& Massarini, A. (2008). Curtis Biología. Madrid: Editorial médica Panamericana.

Flores, J., Caballero, M., \& Moreira, M. (2009). El laboratorio en la enseñanza de las ciencias: Una visión integral en este complejo ambiente de aprendizaje. Revista de Investigación, 33(68), 75-111. 
Bio - grafia. Escritos sobre la Biología y su Enseñanza. ISSN 2027

Edición Extraordinaria. p.p. 798- 811

Memorias del VIII Encuentro Nacional de Experiencias en Enseñanza de la Biología y la Educación Ambiental. III Congreso Nacional de Investigación en Enseñanza de la Biología.

González, J. L. (1995). La paradoja Harvey-Descartes y el proyecto de una biología geométrica. En Arana, J. (Ed.) La filosofía de los científicos (62-82). Sevilla; Universidad de Sevilla- Fundación El Monte.

Jaime, J. C. (2009). Breve historia de la hematología V: La transfusión sanguínea y el trasplante de células hematopoyéticas. En Jaime, J.C. \& Gómez, D. Hematología, La sangre y sus enfermedades (195-204). México: MCGRAW-HILL INTERAMERICANA EDITORES, S.A. de C.V.

Jiménez, M. P. (2003). La enseñanza y el aprendizaje de la biología. En Jiménez, M.P (Ed.), Enseñar ciencias (119-146). Barcelona: Grao.

Medina, E. (1983). La polémica internalismo/externalismo. REIS, (23)53-75. Recuperado de: http://dialnet.unirioja. es/servlet/articulo? codigo= 250835

Lombardi, O. (1997). La pertinencia de la historia en la enseñanza de las ciencias. Enseñanza de las ciencias, 15(3), 343-349.

López, A., \& Tamayo, O. (2012). Las Prácticas de Laboratorio en la Enseñanza de las Ciencias Naturales. Revista Latinoamericana de Estudios Educativos, 8(1),145-166.

Pozo, J. I. (1997). Enfoque para la Enseñanza de las Ciencias. En J. I. Pozo (Ed.), Teorías Cognitivas del Aprendizaje (265-308). Madrid: Morota. 